\title{
Minimally invasive tumor bed implant (MITBI) and peri-operative high-dose-rate brachytherapy (PHDRBT) for accelerated minimal breast irradiation (AMBI) or anticipated boost (A-PHDRBT-boost) in breast-conserving surgery for ductal carcinoma in situ
}

\author{
Marta Gimeno Morales, MD!, Fernando Martinez-Regueira, MD², Natalia Rodriguez-Spiteri, MD², \\ Begoña Olartecoechea, MD², Isabel Rubio, MD², Antonio Esgueva, MD², Luis Pina, MD³, Arlette Elizalde, MD³, \\ Carolina Sobrido Sampedro, MD³, Miguel Angel ldoate, MD², Marta Abengozar, MD4, Luis Ramos, PhD' \\ Felipe Calvo Manuel, MD', Rafael Martinez-Monge, MD', Mauricio Cambeiro, MD'
}

'Department of Oncology, University of Navarra, Pamplona-Madrid, Spain, ${ }^{2}$ Breast Surgical Oncology Unit, University of Navarra, PamplonaMadrid, Spain, ${ }^{3}$ Department of Radiology, University of Navarra, Pamplona-Madrid, Spain, ${ }^{4}$ Department of Pathology. University of Navarra, Pamplona-Madrid, Spain

\begin{abstract}
Purpose: To evaluate our institutional experience of minimally invasive tumor bed implantation (MITBI) during breast-conserving surgery (BCS) for ductal carcinoma in situ (DCIS) to deliver peri-operative high-dose-rate brachytherapy (PHDRBT) as accelerated minimal breast irradiation (AMBI) or anticipated boost (A-PHDRBT-boost).

Material and methods: Patients older than 40 , with clinical and radiological unifocal DCIS $<3 \mathrm{~cm}$ were considered potential candidates for accelerated partial breast irradiation (APBI) and were implanted during BCS using MITBItechnique. Patients who in final pathology reports showed free margins and no other microscopic tumor foci, received AMBI with PHDRBT (3.4 Gy BID in 5 days). Patients with adverse features received A-PHDRBT-boost with post-operative external beam radiotherapy (EBRT).

Results: Forty-one patients were implanted, and 36 were treated and analyzed. According to final pathology, $24(67 \%)$ patients were suitable for AMBI and $12(33 \%)$ were qualified for A-PHDRBT-boost. Reoperation rate for those with clear margins was $16.6 \%$ (6/36); this rate increased to 33\% (4/12) for G3 histology, and 66\% (4/6) were rescued using AMBI. Early complications were documented in 5 patients (14\%). With a median follow-up of 97 (range, 42-138) months, 5-year rates of local, elsewhere, locoregional, and distant control were all 97.2\%. 5-year ipsilateral breast tumor recurrence rates (IBTR) were 5.6\% (2/36), 8.3\% (2/24) for AMBI, and 0\% (0/12) for A-PHDRBT-boost patients. Both instances of IBTR were confirmed G3 tumors in pre-operative biopsies; no IBTR was documented in G1-2 tumors. Cosmetic outcomes were excellent/good in $96 \%$ of AMBI vs. $67 \%$ in A-PHDRBT-boost $(p=0.034)$.

Conclusions: The MITBI-PHDRBT program allows selection of patients with excellent prognoses (G1-2 DCIS with negative margins and no multifocality), for whom AMBI could be a good alternative with low recurrence rate, decrease of unnecessary radiation, treatment logistics improvement, and over-treatment reduction. Patients whose pre-operative biopsy showed G3 tumor, presents with inferior local control and more risk of reoperation due to positive margins.

J Contemp Brachytherapy 2020; 12, 6: 521-532 DOI: https://doi.org/10.5114/jcb.2020.101684
\end{abstract}

Key words: partial breast irradiation, DCIS, high-dose-rate brachytherapy. 


\section{Purpose}

Currently, the standard treatment for ductal breast carcinoma in situ (DCIS) of less than $2-3 \mathrm{~cm}$ is conventional breast-conserving treatment (BCT) with external radiotherapy of the whole breast (whole breast irradiation - WBI). BCT reduces the risk of local ipsilateral breast tumor recurrence (IBTR) by approximately $50 \%$ compared to results with exclusive breast-conserving surgery (BCS): $8.9 \%$ recurrence rate at 15 years for $\mathrm{BCT}$ compared to $19.4 \%$ for exclusive BCS [1]. The benefit of BCT in local control is confirmed when analyzing different subgroups (age, grade, type, and clinical presentation), but has no influence on general or specific survival [2]. Local control mediated by BCT is influenced by several factors, such as resection margins, age, grade, presence of necrosis, and tumor multifocality. Of these, resection margins have the largest influence, providing almost $70 \%$ reduction in the risk of recurrence, with free margins of at least $2 \mathrm{~mm}[3,4]$. Another factor to consider with BCT in DCIS is tumor multifocality, since it predicts the risk of residual disease after BCS [5].

Although in DCIS, the protective effect of adjuvant WBI after BCS is sufficiently documented [6,7], its systematic administration is still controversial, because the observed benefit is not uniform, does not improve survival, and involves administration of moderately high doses of radiation to large volumes of healthy tissue (lung, heart, and the remaining healthy breast without risk). Therefore, WBI in the conservative management of DCIS is considered an over-treatment in many cases $[8,9,10]$. Another limitation of conventional BCT is the duration of WBI (5 weeks), which can pose a logistic challenge for patients.

In selected patients with early breast cancer, accelerated partial breast irradiation (APBI) has become a standard post-operative treatment, since it is an alternative to standard BCT and has similar outcomes, while significantly reducing the duration of treatment and radiation exposure to organs at risk (OARs). A number of single-institution, balloon-based brachytherapy studies $[11,12,13,14,15,16]$ confirmed that this treatment modality achieves adequate IBRT rates, good clinical, and cosmetic outcomes. Consequently, ASTRO guidelines include DCIS patients ( $\geq 50$ years, screen-detected, low- to intermediate-risk, size $\leq 25 \mathrm{~mm}$, and resected margins $\geq 3 \mathrm{~mm}$ ) as suitable candidates for APBI [17].

To reduce the impact of over-treatment, and to overcome some of disadvantages of conventional BCT (radiation in healthy tissues and logistic problems associated with the duration of WBI), our institution launched a minimally invasive tumor bed implant (MITBI) program in 2008 to administer peri-operative high-dose-rate brachytherapy (PHDRBT).

This program uses an initial classification of the risk of recurrence based on definitive pathological data (margins/multifocality) to adapt the volume and intensity of adjuvant radiotherapy to the risk of each patient. Thus, in low-risk patients, accelerated minimal breast irradiation (AMBI) will be administer by exclusive PHDRBT, and in high-risk patients, early boost of the tumor bed (anticipat- ed PHDRBT boost [A-PHDRBT boost]) will be performed in combination with WBI. The endpoint of this study was to evaluate the safety, efficacy, cosmetic results, and toxicity of the MITBI and PHDRBT (AMBI/A-PHDRBTboost) program for DCIS.

\section{Material and methods}

\section{Objectives of the MITBI and PHDRBT program (AMBI/A-PHDRBT-boost)}

Primary objectives:

1. To optimize the recognition of radiation target by intraoperatively distinguishing the tumor bed within the surgical bed.

2. To perform a MITBI, rather than implanting the entire surgical bed.

3. To classify the risk of a relapse according to the status of margins and signs of tumor multifocality in the definitive study of a surgical specimen.

Secondary objectives:

1. To optimize the use of adjuvant radiation by performing accelerated minimal breast irradiation (AMBI) as an exclusive treatment in low-risk patients, or as early boost to the tumor bed (HDR anticipated boost) prior to hypofractionated external radiation therapy in high-risk patients.

2. To benefit the technical performance of multicatheter interstitial brachytherapy (stability of the applicator in the target and high dosimetric quality derived from a 3D planning by image-guided brachytherapy) that are especially attractive for treating small volume tumors.

3. To reduce radiation exposure of healthy tissue (lung, heart, and breast), decrease the risk of long-term toxicity, and improve cosmetic results in low-risk patients.

4. To improve the logistics of treatment, especially in low-risk patients treated with AMBI, by significantly decreasing the duration of locoregional treatment to 11 days (from surgery) compared to 60-70 days required for conventional BCT.

\section{Inclusion criteria for MITBI and AMBI}

Patients older than 40 , with a vacuum-assisted biopsy diagnosis of DCIS that was less than $3 \mathrm{~cm}$ in size and confirmed by mammogram, ultrasound, and/or magnetic resonance imaging (MRI) as well as by surgeon's criteria to be unifocal, were included in the study. The institutional board approved prospective recruitment for the study. All patients signed a consent form.

\section{MITBI procedure}

Usually, lumpectomy is performed through skin over the tumor. Once the lumpectomy is performed, the tumor bed must be recognized within the surgical bed. Tumor bed is the target of implant and corresponds to the projection zone of tumor on both cavity walls (marked with four titanium clips apart), surrounded with 1-2 cm (Figure $1 C, D)$. The definition of the tumor bed is determined on the base of topographical information in pre-operative 
breast radiology imaging, information from clinical surgeon, and examination of surgical specimen.

In the present study, the surgical clip zone and a margin of 1-2 cm was completely encompassed by the implant. Implantation was performed intraoperatively by freehand technique with sharp needles (Nucletron, Elekta $\mathrm{AB}$, Stockholm, Sweden), and in all cases, the needles were placed perpendicularly to the axis to avoid geometrical disturbances and to promote wound-closure (Figure 1D). The implant extended from the depth to the surface and from the inside to the outside; the intraplane catheters
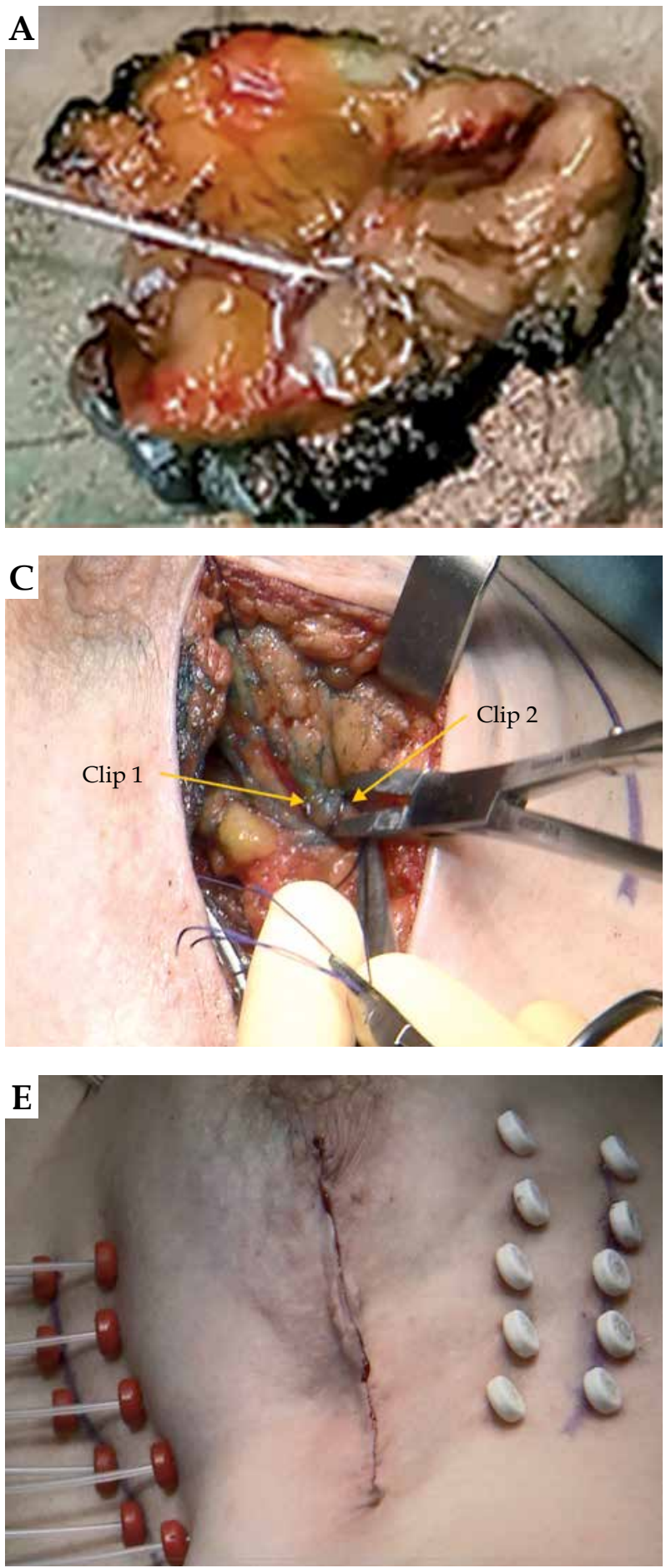

were placed 1-1.5 cm apart, following Paris system recommendations. All patients received prophylactic oral antibiotics.

Regarding margin status on final pathology after surgery, reoperation indication was established by tumor on ink or close margin (less than $2 \mathrm{~mm}$ ) on G3 histology.

\section{MITBI-AMBI protocol treatment}

Computed tomography planning was performed 48 hours after surgery and MITBI. Clinical target volume
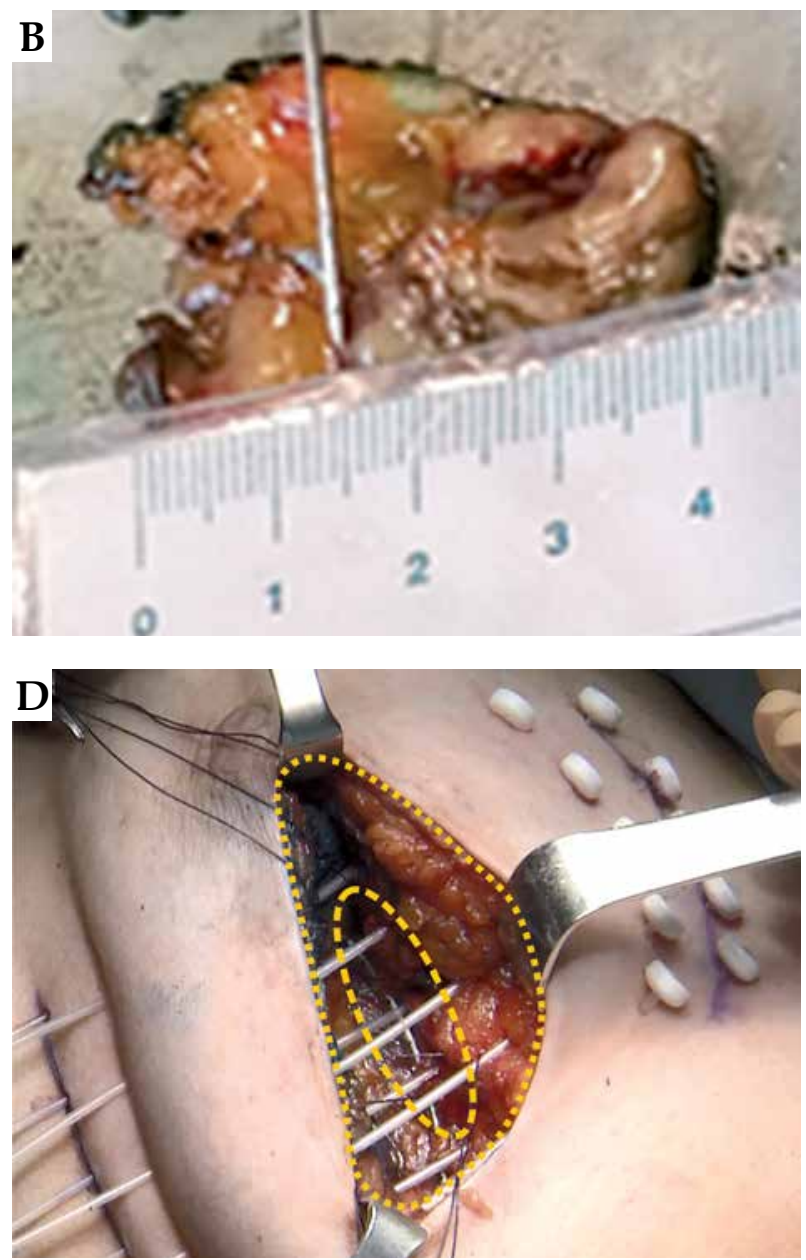

Fig. 1. Minimally invasive tumor breast implant (MITBI). A) Tumor on surgical specimen, B) measurement tumor to margin, C) two of four titanium clips marking the projection of the tumor on superior wall of the surgical bed, D) tumor bed (yellow line) inside surgical bed (orange point line). Tumor bed include clips' zone and $1-2 \mathrm{~cm}$ surrounding of breast tissue encompassed by the catheters 
(CTV) was contoured from the recognition of clip zone and surrounding 1-2 cm encompassed by the catheters.

Based on definitive pathological results, patients with pure DCIS, tumor size $<3 \mathrm{~cm}$, and margins $>2 \mathrm{~mm}$ with no signs of microscopic multifocality were classified as low- risk patients, and received $34 \mathrm{~Gy}$ of AMBI delivered in 10 fractions, 6 hours apart ( $3.4 \mathrm{~Gy} /$ fraction), over 5 consecutive days. Patients implanted with positive or very close margins not suitable for additional wide excision, or those with pathological microscopic multifocality classified as high-risk patients, received A-PHDRBT-boost (13.6 Gy in 4 fractions, 6 hours apart; 3.4 Gy/fraction over 2 consecutive days), followed by 39.9 Gy of hypofractionated whole breast irradiation in 15 fractions (START B), over 3 weeks after brachytherapy procedure.

The dosimetric parameters were defined as: CTV $\mathrm{D}_{90}$ coverage $>90 \%$, dose homogeneity index $(\mathrm{DHI})>0.7$, and the skin $\mathrm{D}_{10}<70 \%$. New GEC-ESTRO interstitial multicatheter APBI recommendations [18] were calculated retrospectively and analyzed. Patients with positive estrogen receptors received a hormonal treatment.

\section{Follow-up and clinical outcomes}

Follow-up included clinical examination by breast surgeons and radiation oncologists at 1 month after the brachytherapy procedure and every 6 months thereafter for 5 years. Mammogram and breast ultrasound were done annually. Ipsilateral breast tumor recurrence was classified as true failure (TF) or elsewhere failure (EF), according to clinical site in relation to the initial tumor bed. TF was defined as a recurrence within or immediately adjacent to the primary tumor site or irradiated area. EF was classified as an ipsilateral breast recurrence several centimeters from the primary site or in other breast quadrant. Local control was established when no IBRT was documented. Regional and distant metastasis were also recorded.

\section{Early and late complications, acute and late toxicity, radiation-related effects, cosmetic evaluation, and radiologic findings}

All early or late complications were recorded in an electronic chart. Acute and late toxicities were graded according to the Radiation Therapy Oncology Group and the European Organization for Research and Treatment of Cancer (RTOG/EORTC) radiation morbidity scoring scheme [19]. Radiation-related effects were categorized as: breast symmetry (symmetry, acceptable difference, obvious difference, or marked difference), breast retraction (not visible, slightly visible, obvious, or marked), hyperpigmentation (not visible, slightly visible, obvious, or marked), catheter puncture marks (not visible, slightly visible, obvious, or marked), skin telangiectasia (not visible, slightly visible, obvious, or marked), and skin-subcutaneous atrophy or fibrosis (absence, light, moderated, or severe). Cosmetic evaluation was based on Wazer's criteria: excellent, good, fair, or poor [20]. The presence of oil-cyst, fat necrosis, and symptomatic fat necrosis were assessed by a mammogram, ultrasound, and physical examination during follow-up. Data were collected and recorded at every clinical visit.

\section{Statistical analysis}

Univariate analysis of different clinical and treatment quantitative variables included its central tendency (percentages, mean, or median) or dispersion (range). Discrete variables were compared using Fisher's exact test method and $\chi^{2}$ test, with $p<0.05$ considered as statistically significant. Continuous variables were compared using Wilcoxon-Mann-Whitney $U$ test $(p<0.05$ statistical significance). Kaplan-Meier method was applied to calculate survival results from the date of surgery to the last follow-up visit. All the statistical analysis was performed with IBM SPSS Statistics 20 (IBM Corp., USA).

\section{Results}

Between November 2008 and October 2016, 44 patients were initially evaluated in the program as candidates for an implant and AMBI. One patient refused to participate, and 2 patients were excluded intraoperatively due to anatomical limitations (surgical cavity located very eccentrically in the inner superior quadrant, with very thin breast tissue). Among the 41 patients implanted, 4 were further excluded after definitive pathology reporting infiltrating carcinoma. Moreover, one patient refused reimplantation during reoperation; this patient was excluded from the analysis. In total, 36 patients consisted a cohort of the study. Pre-operative patient characteristics are described in Table 1.

\section{MITBI-AMBI protocol treatment}

Definitive pathological findings showed that 24 patients $(67 \%)$ met the criteria for AMBI, and 12 patients (33\%) for A-PHDRBT-boost. Reasons for anticipated boost were close margins in 4 patients (33\%), multifocality in 6 patients $(50 \%)$, and both adverse pathological features in 2 patients (16\%). Table 2 shows definitive pathological and surgical characteristics. Median time from an implant to brachytherapy was 6 days (range, 2-9 days).

\section{Reoperation due to margin status}

Six patients $(16.6 \%)$ underwent re-excision for positive margins or presence of G3 DCIS less than $2 \mathrm{~mm}$ from the margin. Among these, 4 patients (66\%) had a G3 DCIS. During the re-excision, the implant was replaced in 6 patients, $4(66 \%)$ underwent AMBI, and $2(33 \%)$ a boost. Details are shown in Table 2.

\section{Reoperation due to bleeding}

Three patients $(8 \%)$ presented complications related to bleeding. Two were resolved with compressive measures, and one required surgical wound revision to confirm whether the cause of bleeding was related to placement of the catheter.

\section{Dosimetric characteristics}

Dosimetric parameters are described in Table 3. New GEC-ESTRO practical recommendations for multicatheter breast implant were included and reported. OARs 
Table 1. Pre-operative patients' characteristics according to treatment protocol

\section{Characteristic}

Patient and treatment

Age, median (range)

Hormonal status

Peri-menopausal

Pre-menopausal

Type of tumor

Unilateral primary

Bilateral primary

Contralateral primary

Type of implant

Bilateral
Unilateral
Breast side
Right breast

\begin{tabular}{ll}
\hline Right breast & 20 \\
\hline Left breast & 16 \\
Mammography findings
\end{tabular}

$n(\%)$

$36(100)$

$56(41-77)$

$3(8.3)$

$22(61.1)$

$11(30.6)$

$32(88.9)$

$2(5.6)$

$2(5.6)$

$2(5.6)$

$34(94.4)$

20 (55.6)

$16(44.4)$

$34(94.4)$

2 (5.6)

No

Ultrasound

Yes $12(33.3)$

No $16(44.4)$

Not performed

$8(22.2)$

MRI diagnosis

Yes
No

Not performed

Multifocality

Multicentricity

$4(66.7)$

7 (19.4)

5 (13.9)

$0(0)$

$0(0)$

Radiologic tumor size (cm)

Mean
Median
Range
Grade

\begin{tabular}{lccc}
\hline G1 & $10(27.8)$ & $7(29.2)$ & $3(25)$ \\
\hline $\mathrm{G} 2$ & $13(36.1)$ & $8(33.3)$ & $5(41.6)$ \\
\hline $\mathrm{G} 3$ & $12(33.3)$ & $8(33.3)$ & $4(33.3)$ \\
\hline Not described & $1(2.8)$ & $1(4.4)$ & $0(0)$ \\
\hline Hormonal receptor (ER and PR) & & $12(50)$ & $5(41.6)$ \\
\hline Positive & $17(47.2)$ & $0(0)$ & $1(8.3)$ \\
\hline Negative & $1(2.8)$ & $12(50)$ & $6(50)$
\end{tabular}

$A M B I$ - accelerated minimal breast irradiation, A-PHDRBT-boost - anticipated peri-operative high-dose-rate brachytherapy boost, MRI - magnetic resonance imaging, ER - estrogen receptor, $P R$ - progesterone receptor

$\mathrm{AMBI}, n(\%)$

A-PHDRBT-boost, $n(\%)$

24 (67)

12 (33)

52 (41-76)

59 (41-77)

1 (8.3)

5 (41.6)

$6(50)$

9 (75)

$1(8.3)$

1 (4.4)

2 (16.6)

0 (0)

1 (8.3)

11 (91.6)

$23(95.8)$

$6(50)$

$6(50)$

$10(41.6)$

11 (91.6)

$1(8.3)$

$1(4.4)$

$7(58.3)$

$5(20.8)$

$4(33.3)$

7 (29.2)

$1(8.3)$

1.3

1.2

1.5

10.8

1.1

0.5-3

$0.4-3$

\begin{tabular}{ll}
1 & $0.4-3$ \\
\hline & $0.4-3$
\end{tabular}

8 (66.6)

2 (16.6)

2 (16.6)

$0(0)$

0 (0)

0.8

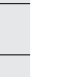


Table 2. Definitive, pathological, and surgical characteristics

\begin{tabular}{|c|c|c|c|}
\hline Features & Total, $n(\%)$ & $\mathrm{AMBI}, n(\%)$ & A-PHDRBT-boost, $n(\%)$ \\
\hline Patients' distribution & $36(100)$ & $24(67)$ & $12(33)$ \\
\hline \multicolumn{4}{|l|}{ Pathological tumor size (cm) } \\
\hline Mean & 1.2 & 1.2 & 1.3 \\
\hline Median & 1 & 1 & 1.2 \\
\hline Range & $0.2-3$ & $0.2-2.5$ & $0.5-3$ \\
\hline \multicolumn{4}{|l|}{ Tumor size $(\mathrm{cm})$} \\
\hline$\leq 1$ & $18(50)$ & $13(54.2)$ & $5(41.6)$ \\
\hline$\geq 1.1-\leq 2$ & $15(41.7)$ & $9(37.5)$ & $6(50)$ \\
\hline$\geq 2.1-\leq 3$ & $3(8.3)$ & $2(8.3)$ & $1(8.3)$ \\
\hline \multicolumn{4}{|l|}{ Pathological microscopic multifocality } \\
\hline Yes & $7(19.4)$ & $0(0)$ & $7(58.3)$ \\
\hline No & $29(80.6)$ & $24(100)$ & $5(41.6)$ \\
\hline \multicolumn{4}{|l|}{ Margins status } \\
\hline On ink & $1(2.8)$ & $0(0)$ & $1(8.3)$ \\
\hline Close $(<2 \mathrm{~mm})$ & $7(19.4)$ & $2(8.3)$ & $5(41.6)$ \\
\hline Free $(>2 \mathrm{~mm})$ & $28(77.8)$ & $22(91.6)$ & $6(50)$ \\
\hline \multicolumn{4}{|l|}{ Necrosis } \\
\hline Yes & $10(27.8)$ & $7(29.2)$ & $3(25)$ \\
\hline No & $26(72.7)$ & $17(70.8)$ & $9(75)$ \\
\hline \multicolumn{4}{|l|}{ Grade } \\
\hline G1 & $7(19.4)$ & $4(16.6)$ & $3(25)$ \\
\hline G2 & $14(38.9)$ & $9(37.5)$ & $5(41.6)$ \\
\hline G3 & $15(41.7)$ & $11(45.8)$ & $4(33.3)$ \\
\hline \multicolumn{4}{|l|}{ Hormonal receptor (ER and PR) } \\
\hline Positive & $28(77.8)$ & $19(79.2)$ & $9(75)$ \\
\hline Negative & $8(22.2)$ & $5(20.8)$ & $3(25)$ \\
\hline \multicolumn{4}{|l|}{ Van Nuis PI aged modify } \\
\hline $4-6$ & $20(55.6)$ & $14(58.3)$ & $6(50)$ \\
\hline $7-9$ & $15(41.7)$ & $10(41.6)$ & $5(41.6)$ \\
\hline $10-12$ & $1(2.8)$ & $0(0)$ & $1(8.3)$ \\
\hline \multicolumn{4}{|l|}{ Type of surgery } \\
\hline Lumpectomy & $22(61)$ & $15(62.5)$ & $7(58.3)$ \\
\hline Lumpectomy and sentinel node & $14(39)$ & $9(37.5)$ & $5(41.6)$ \\
\hline Reoperation due to affected or close margins & $6(16)$ & $4(16.6)$ & $2(16)$ \\
\hline G1 & $1(16)$ & $0(0)$ & $1(8.3)$ \\
\hline G2 & $1(16)$ & $1(4.2)$ & $0(0)$ \\
\hline $\mathrm{G} 3$ & $4(66)$ & $3(12.5)$ & $1(8.3)$ \\
\hline
\end{tabular}

$A M B I$ - accelerated minimal breast irradiation, A-PHDRBT-boost - anticipated peri-operative high-dose-rate brachytherapy boost, ER - estrogen receptor, PR - progesterone receptor 
constraints (skin, heart, lung, adjacent normal tissue, and rib), planning target volume (PTV), and implant parameters (DHI, dose nonuniformity ratio - DNR, and conformal index - COIN) were adequately respected and covered.

\section{Complication, toxicity, and radiological findings during follow-up}

Early complications, acute toxicity, late toxicity, and radiation-related effects were evaluated in all the patients. The relevant data are described in Table 4. All patients in the AMBI group and $92 \%$ of patients in the boost group developed acute grade $\leq 2$ toxicities. One patient in the boost group experienced a major acute RTOG grade 3 or higher toxicity, resulting in a bad cosmetic outcome. All late toxicities described in the study were grade $\leq 2$. No major late grade 3, or higher RTOG skin or subcutaneous toxicities were documented in any of the groups. Moreover, no statistical differences related to toxicity were found between the groups.

Radiation-related effects, symmetry, retraction, hyperpigmentation, catheter puncture marks, skin telangiectasia, skin-subcutaneous atrophy, and fibrosis were assessed in all the patients. Although, these effects were not visible or were slightly noticeable in a percentage of the patients in both groups, worse symmetry $(p=0.021)$ and retraction $(p=0.001)$ were statistically significant in the A-PHDRBT-boost group compared to results in the AMBI group.

\section{Radiological findings during follow-up and cosmetics outcomes}

On radiological follow-up, oil cysts developed in 12 patients $(33 \%)$, while radiologic fat necrosis developed in $11(30 \%)$ cases, out of which only $1(2.8 \%)$ was symptomatic and required surgical removal. No statistical differences were found between the groups regarding radiological findings.

Cosmetic outcomes in AMBI patients were excellent or good in 23 patients $(95.8 \%)$, and fair or poor in one patient $(4.2 \%)$. Cosmetic outcomes in A-PHDRBT-boost patients were excellent or good in 8 patients $(67 \%)$, and fair in 4 patients $(33 \%)$. The difference between the groups was not statistically significant $(p=0.034)$. Further information on all categories are described in Table 5.

\section{Clinical outcomes}

The median follow-up for all patients was 97 (range, 42-139) months. For the AMBI group, the median follow-up was 100 (range, 43-137) months, and for A-PHDRBT-boost patients, 89 (range, 42-119) months.

For all patients, the rate of local, elsewhere, locoregional, and distant control was $97.2 \%$. For the AMBI group $(n=24)$, the rates of local, elsewhere, locoregional, contralateral, and distant control were $95.8 \%, 95.8 \%$, $100 \%, 100 \%$, and $100 \%$, respectively. One true local recurrence and one elsewhere were reported in this group at 62.2 and 103 months of follow up, respectively; both cases were G3 DCIS. The cumulative 5-year incidence of IBTR
Table 3. Dosimetry

Features

\begin{tabular}{lc} 
Features & Results \\
\hline Type of implant & \\
\hline Two planes, $n(\%)$ & $32(89)$ \\
\hline Three planes, $n(\%)$ & $4(11)$ \\
\hline Number of catheters & \\
\hline Median (range) & $8(5-14)$ \\
\hline Time surgery to HDR-BT &
\end{tabular}

Time surgery to HDR-BT

treatment

\begin{tabular}{lc}
\hline Median (range) & $6(2-9)$ \\
\hline $\begin{array}{l}\text { Breast (cc) } \\
\text { Median (range) }\end{array}$ & $671(346-1257)$ \\
\hline CTV (cc) & $37.4(13-70)$ \\
\hline Median (range) & $44(16-112)$ \\
\hline$V_{100}(c c)$ & \\
\hline Median (range) & $0.72(0.5-0.8)$ \\
\hline \begin{tabular}{l} 
DHI CTV \\
\hline Median (range)
\end{tabular} \\
\hline \begin{tabular}{l} 
DHI volume external \\
\hline Median (range)
\end{tabular} \\
\hline \begin{tabular}{l} 
DNR \\
\hline Median (range)
\end{tabular} \\
\hline \begin{tabular}{l} 
COIN \\
\hline Median (range)
\end{tabular} \\
\hline OARs constraints ${ }^{1}$ & $0.25(0.2-0.4)$ \\
\hline Organ & AMBI \\
\hline
\end{tabular}

\begin{tabular}{|c|c|c|}
\hline Organ & $\mathrm{AMBI}$ & A-PHDRBT-boost \\
\hline \multicolumn{3}{|c|}{ Skin (Gy) (\% of PD) } \\
\hline Median $\mathrm{D}_{10}$ & $18.7(54)$ & $7.2(55)$ \\
\hline Median $\mathrm{D}_{1 \mathrm{cc}}$ & $21.5(63)$ & $8.4(62)$ \\
\hline Median $\mathrm{D}_{0.2 c c}$ & $24.4(72)$ & $10.1(74)$ \\
\hline \multicolumn{3}{|l|}{$\operatorname{Rib}(\mathrm{Gy})(\%$ of PD) } \\
\hline Median $\mathrm{D}_{1 \mathrm{cc}}$ & $11.8(35)$ & $6.5(48)$ \\
\hline Median $\mathrm{D}_{0.1 \mathrm{cc}}$ & $14.3(42)$ & $8.1(60)$ \\
\hline \multicolumn{3}{|c|}{ Breast no target (\%) } \\
\hline Median $\mathrm{V}_{90}$ & 3.1 & 3.7 \\
\hline Median $V_{50}$ & 13.7 & 13.6 \\
\hline \multicolumn{3}{|c|}{ Lung (Gy) (\% of PD) ${ }^{2}$} \\
\hline Median MLD & $1.13(3.3)$ & 7.27 \\
\hline Median $\mathrm{D}_{0.1 \mathrm{cc}}$ & $10.8(32)$ & 40 \\
\hline \multicolumn{3}{|c|}{ Heart (Gy) $(\% \text { of PD) })^{2}$} \\
\hline Median MHD & $0.93(3)$ & 1.8 \\
\hline Median $\mathrm{D}_{0.1 \mathrm{cc}}$ & $4.5(13)$ & 18 \\
\hline
\end{tabular}

${ }^{1}$ some constraint values are presented as absolute dose (Gy) and as relative percentage of prescription dose, following the GEC-ESTRO recommendations [18], ${ }^{2}$ exclusively for lung and heart constraint values, we also calculated the EBRT dose received

HDR-BT - high-dose-rate brachytherapy, CTV - clinical target volume, DHI dose homogeneity index, DNR - dose nonuniformity ratio, COIN - conformal index, OARs - organs at risk, PD - prescription dose, MLD - mean lung dose, $M H D$ - mean heart dose 
Table 4. Early and late complications, and Radiation Therapy Oncology Group (RTOG) toxicity

\begin{tabular}{|c|c|c|c|}
\hline Features & Total, $n(\%)$ & $\mathrm{AMBI}, n(\%)$ & A-PHDRBT-boost, $n(\%)$ \\
\hline Early complications (major and minor) & $5(14)$ & $4(16)$ & $1(8.3)$ \\
\hline Reoperation due to hemorrhage & $1(3)$ & $0(0)$ & $1(8.3)$ \\
\hline Self-limited hemorrhage & $3(8.3)$ & $2(8.3)$ & $1(8.3)$ \\
\hline Wound dehiscence/necrosis & $0(0)$ & $0(0)$ & $0(0)$ \\
\hline Acute seroma & $0(0)$ & $0(0)$ & $0(0)$ \\
\hline Local infection & $2(5.6)$ & $2(8.3)$ & $0(0)$ \\
\hline Skin subcutaneous necrosis & $2(5.6)$ & $2(8.3)$ & $0(0)$ \\
\hline Late complications & $3(8.3)$ & $2(8.3)$ & $1(8.3)$ \\
\hline Seroma & $0(0)$ & $0(0)$ & $0(0)$ \\
\hline Symptomatic fat necrosis & $1(2.8)$ & $1(4.2)$ & $0(0)$ \\
\hline Breast pain & $2(5.6)$ & $1(4.2)$ & $1(8)$ \\
\hline Lymphedema & $0(0)$ & $0(0)$ & $0(0)$ \\
\hline \multicolumn{4}{|l|}{ Acute RTOG skin or subcutaneous toxicity } \\
\hline Grade 0 & $13(36.1)$ & $11(45.8)$ & $2(16.6)$ \\
\hline Grade 1 & $17(47.2)$ & $11(45.8)$ & $6(50)$ \\
\hline Grade 2 & $5(13.9)$ & $2(8.3)$ & $3(25)$ \\
\hline Grade 3 & $1(2.8)$ & $0(0)$ & $1(8.3)$ \\
\hline Grade 4 & $0(0)$ & $0(0)$ & $0(0)$ \\
\hline \multicolumn{4}{|l|}{ Late RTOG skin or subcutaneous toxicity } \\
\hline Grade 0 & $18(50)$ & $14(58.3)$ & $4(33.3)$ \\
\hline Grade 1 & $9(25)$ & $6(25)$ & $3(12.5)$ \\
\hline Grade 2 & $9(25)$ & $4(16.6)$ & $5(41.6)$ \\
\hline Grade 3 & $0(0)$ & $0(0)$ & $0(0)$ \\
\hline Grade 4 & $0(0)$ & $0(0)$ & $0(0)$ \\
\hline
\end{tabular}

AMBI - accelerated minimal breast irradiation, A-PHDRBT-boost - anticipated peri-operative high-dose-rate brachytherapy boost

in the AMBI group was $8.3 \%(2 / 24)$ (Figure 2). Furthermore, based on biopsy tumor grade (G1-2 vs. G3), the failure-free survival rate in the AMBI group tended towards statistically significant ( $p=0.067$ ) (Figure 3). Only one patient in the A-PHDRBT-boost group developed locoregional progression and systemic disease due to previous contralateral ductal infiltrating carcinoma diagnosed 16 years before MITBI for DCIS. All data are presented in Table 6.

\section{Discussion}

The role of APBI has been explored in a variety of prospective studies (ELIOT, TARGIT, IMPORT LOW, Budapest experience, and GEC-ESTRO) [21,22,23,24,25]. In all of these studies, the tissue adjacent to surgical bed was protected using a safety margin of $1-2 \mathrm{~cm}$. In the APBI reference studies, only the GEC-ESTRO study included 35 patients $(5.5 \%)$ with DCIS in the APBI arm, without a control analysis by subgroup [25]. Institutional experiences with Mammosite showed no differences in the rates of IBTR between DCIS and the invasive suitable/cautionary risk group; this therapy also achieved good clinical outcomes, low acute and late toxicity, and acceptable cosmesis [26]. Despite the lack of strong bibliographic evidence, APBI has recently been included in the ASTRO guidelines as a suitable radiotherapy modality for the treatment of G1 and G2 DCIS of less than $2.5 \mathrm{~cm}$, with free margins of more than $3 \mathrm{~mm}$ [17].

Among the different techniques used in the APBI reference studies of early breast cancer, multicatheter interstitial brachytherapy (MBI) offers the best recurrence rate to date, with a 5-year cumulative incidence of local recurrence of $1.4 \%$ [25]. Additionally, MBI offers very high performance in planning and dosimetry, with multiple dwell points throughout the implant, which allow scrupulous modulation, target shaping, and documentation of radiation dose to target and OARs. 
Table 5. Radiation-related effects and cosmetic evaluation

\begin{tabular}{|c|c|c|c|c|}
\hline Features & Total, $n(\%)$ & $\mathrm{AMBI}, n(\%)$ & A-PHDRBT-boost, $n(\%)$ & $\chi^{2}, p$-value \\
\hline Symmetry & & & & 0.021 \\
\hline Symmetry & $19(52.8)$ & $15(62.5)$ & $4(33.3)$ & \\
\hline Acceptable difference & $11(30.6)$ & $8(33.3)$ & $3(25)$ & \\
\hline Obvious difference & $2(5.6)$ & $1(4.16)$ & $1(8.3)$ & \\
\hline Marked difference & $4(11.1)$ & $0(0)$ & $4(33.3)$ & \\
\hline Retraction & & & & 0.001 \\
\hline Not visible & $28(77.8)$ & $23(95.8)$ & $5(41.6)$ & \\
\hline Slightly visible & $5(13.9)$ & $1(4.16)$ & $4(33.3)$ & \\
\hline Obvious & $3(8.3)$ & $0(0)$ & $3(25)$ & \\
\hline Marked & $0(0)$ & $0(0)$ & $0(0)$ & \\
\hline Hyperpigmentation & & & & 0.345 \\
\hline Not visible & $19(52.8)$ & $14(58.3)$ & $5(41.6)$ & \\
\hline Slightly visible & $17(47.2)$ & $10(41.6)$ & $7(58.3)$ & \\
\hline Obvious & $0(0)$ & $0(0)$ & $0(0)$ & \\
\hline Marked & $0(0)$ & $0(0)$ & $0(0)$ & \\
\hline Catheter puncture marks & & & & 0.745 \\
\hline Not visible & $21(58.3)$ & $13(54.1)$ & $8(66.6)$ & \\
\hline Slightly visible & $12(33.3)$ & $9(37.5)$ & $3(25)$ & \\
\hline Obvious & $3(8.3)$ & $2(8.3)$ & $1(8.3)$ & \\
\hline Marked & $0(0)$ & $0(0)$ & $0(0)$ & \\
\hline Skin telangiectasia & & & & 0.531 \\
\hline Not visible & $32(88.9)$ & $21(87.5)$ & $11(91.6)$ & \\
\hline Slightly visible & $2(5.6)$ & $1(4.1)$ & $1(8.3)$ & \\
\hline Obvious & $2(5.6)$ & $2(8.3)$ & $0(0)$ & \\
\hline Marked & $0(0)$ & $0(0)$ & $0(0)$ & \\
\hline Skin-subcutaneous atrophy or fibrosis & & & & 0.589 \\
\hline Not visible & $17(47.2)$ & $11(45.8)$ & $6(50)$ & \\
\hline Slightly visible & $17(47.2)$ & $11(45.8)$ & $6(50)$ & \\
\hline Obvious & $2(5.3)$ & $2(8.3)$ & $0(0)$ & \\
\hline Marked & $0(0)$ & $0(0)$ & $0(0)$ & \\
\hline Oil cyst & $12(33)$ & $8(33.3)$ & $4(33.3)$ & \\
\hline Fat necrosis & $11(30)$ & $7(29.2)$ & $4(33.3)$ & \\
\hline Symptomatic fat necrosis & $1(2.8)$ & $1(4.2)$ & $0(0)$ & \\
\hline \multicolumn{5}{|l|}{ Cosmetic outcomes } \\
\hline Wazer's criteria & & & & 0.022 \\
\hline Excellent & $22(61.1)$ & $18(75)$ & $4(33.3)$ & \\
\hline Good & $9(25)$ & $5(20.8)$ & 4 (33.3) & \\
\hline Fair & $5(13.9)$ & $1(4.1)$ & $4(33.3)$ & \\
\hline Poor & $0(0)$ & $0(0)$ & $0(0)$ & \\
\hline
\end{tabular}

AMBI - accelerated minimal breast irradiation, A-PHDRBT-boost - anticipated peri-operative high-dose-rate brachytherapy boost 


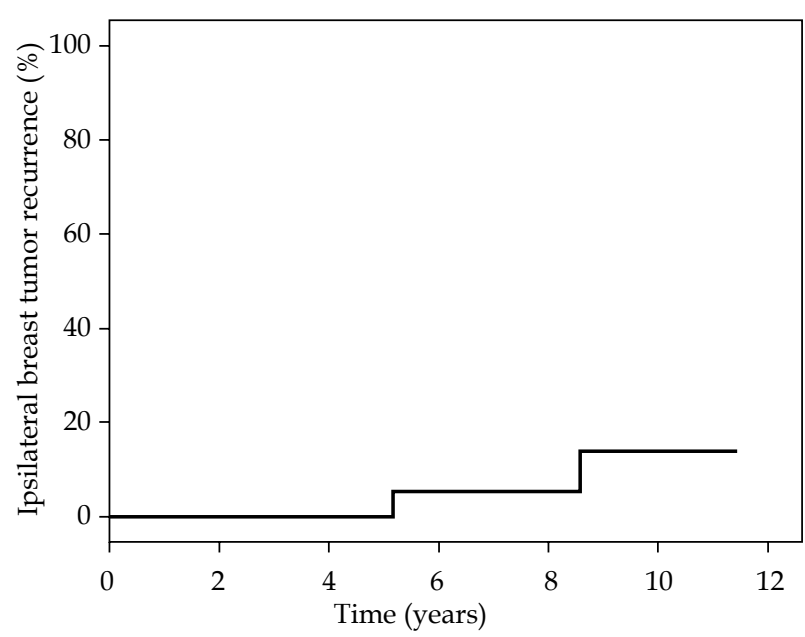

Fig. 2. Ipsilateral breast tumor recurrence (IBTR) incidence in accelerated minimal breast irradiation (AMBI) group

Taking into account the strengths of the MBI technique (good recurrence rates and excellent dosimetric quality), we intended to use AMBI to enhance the effectiveness of adjuvant radiotherapy by reducing the volume treated to only the tumor bed with margin instead of the surgical bed with margin, as in the usual procedure with APBI techniques. Unlike post-operative interstitial multicatheter techniques, $\mathrm{AMBI}$ requires the tumor bed

Table 6. Clinical outcomes by a grade

\begin{tabular}{|c|c|c|c|}
\hline & Total (\%) & AMBI (\%) & $\begin{array}{l}\text { A-PHDRBT } \\
\text { boost (\%) }\end{array}$ \\
\hline \multicolumn{4}{|l|}{ Recurrences rates } \\
\hline Local recurrence (TR) & 2.7 & 4.1 & 0 \\
\hline G1-G2 & 0 & 0 & 0 \\
\hline $\mathrm{G} 3$ & 8.3 & 12.5 & 0 \\
\hline IBTR & 5.6 & 8.3 & 0 \\
\hline G1-G2 & 0 & 0 & 0 \\
\hline G3 & 16 & 25 & 0 \\
\hline \multicolumn{4}{|l|}{ Control rates } \\
\hline Local control & 97.2 & 95.8 & 100 \\
\hline G1-G2 & 100 & 100 & 100 \\
\hline G3 & 91.6 & 87.5 & 100 \\
\hline Elsewhere control & 97.2 & 95.8 & 100 \\
\hline G1-G2 & 100 & 100 & 100 \\
\hline $\mathrm{G3}$ & 93.6 & 87.5 & 100 \\
\hline Locoregional control & 97.2 & 100 & 91.6 \\
\hline G1-G2 & 95.8 & 100 & 87.5 \\
\hline G3 & 100 & 100 & 100 \\
\hline Distant control & 97.2 & 100 & 91.6 \\
\hline G1-G2 & 95.8 & 100 & 87.5 \\
\hline $\mathrm{G3}$ & 100 & 100 & 100 \\
\hline
\end{tabular}

AMBI - accelerated minimal breast irradiation, A-PHDRBT-boost - anticipated peri-operative high-dose-rate brachytherapy boost, IBTR - ipsilateral breast tumor recurrence rates

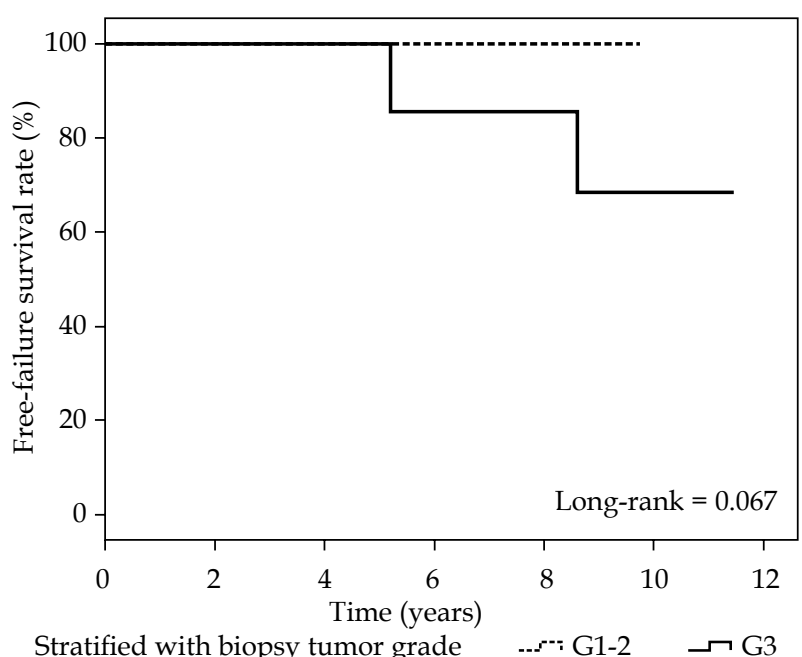

Stratified with biopsy tumor grade

Fig. 3. Disease-free survival in the accelerated minimal breast irradiation (AMBI) group and biopsy grades

to be clearly distinguished from the surgical bed, so that irradiation can be delivered exclusively to the minimal breast tissue at risk after resection, with a maximal stability (interstitial implant), precise target conformation (multicatheter implant/3D dosimetry planning), and high-dose gradient. Finally, this procedure allows to achieve lower implant volumes compared to volumes treated using other devices or post-operative multicatheter APBI techniques.

In the present study, the median follow-up was 97 months (100 months in the AMBI group and 89 in the PHDRBT boost group). The incidence of IBTR in the AMBI group was $8.3 \%(2 / 24)$ and $0 \%(0 / 12)$ in the A-PHDRBTboost group. The cumulative incidence of IBTR at 5 years for the entire study was $5.6 \%(2 / 36)$.

Although the median follow-up of our study was long, these results must be interpreted with caution due to limited number of patients. Nevertheless, the IBTR rate in our study is encouraging compared to 10-year IBTR rates of $15 \%$ that were reported for radiotherapy arms in reference WBI studies (NSABP B-17 and EORTC 10853), and when compared with recurrence rates exceeding $30 \%$ reported for exclusive lumpectomy arms $[27,28]$. Other authors have explored the efficacy of different APBI techniques to treat DCIS. Our 5-year rate of IBRT $(8.3 \%)$ is comparable to IBRT rates reported with balloon-based implants $(0.7 \%$ to $4.1 \%)$ [11,12,13,14,15,16] and with IOERT $(11 \%)$ in patients considered suitable for APBI according ASTRO criteria [29].

In our study, G3 DCIS was associated with a high-rate of reoperation due to involved margins and with a worse proportion of IBTR compared to outcomes reported with standard treatments. Only two patients who developed IBTR in the AMBI group had high-grade tumors (G3); the IBRT rate for the whole group was $16 \%(2 / 12)$ and $25 \%$ $(2 / 8)$ for the AMBI group (Table 6). Additionally, the percentage of reoperation for G3 tumors was high as 33\% $(4 / 12)$. For these reasons, in our institution, we decided that patients with a pre-operative diagnostic of G3 DCIS would not be included in the MITBI and PHDRBT pro- 
gram, but should be considered as candidates for whole breast irradiation.

It is important to note that, in patients with G1-2 tumors, the 5-year local control for the AMBI group was $100 \%$. This finding shows the efficiency and selectivity of AMBI technique, in which the treated volume median $\mathrm{V}_{100}$ was $40 \mathrm{cc}$ and the remaining median breast volume was 671 cc. These data suggest that the MITBI/AMBI combination for patients with low-risk G1-2 tumors significantly optimizes the use of adjuvant radiation by treating less than $10 \%(6.5 \%)$ of the breast, providing $100 \%$ of local control. In this special low-risk group of patients, the RTOG 9804 randomized clinical trial [7] with a median follow-up of 7.2 years reported a $6.7 \%$ IBTR rate without adjuvant radiotherapy, and a $0.9 \%$ IBTR rate for the whole breast radiotherapy arm. This result indicates that AMBI can offer local control, with IBTR rates similar to those of WBI, while minimizing the radiation dose to OARs and normal breast tissue.

This result $\left(\mathrm{V}_{100}\right.$ less than $10 \%$ of breast volume $)$ could also be clinically reflected in our study with cosmetic results and radiation doses to OARs. In AMBI group, $96 \%$ of patients achieved excellent/good cosmetic results compared to $67 \%$ rate in the PHDRBT boost group $(p=0.034)$. Furthermore, the higher BED achieved in the PHDRBT boost group regimen (anticipated boost and EBRT) could compromise the cosmesis. On the other hand, a significant reduction of unnecessary radiation exposure to OARs was documented: lung (median lung dose in $\mathrm{AMBI}=1.13 \mathrm{~Gy}$ vs. median lung dose in A-PHDRBT-boost $=7.27 \mathrm{~Gy}, p=0.0000062$ ) and heart (median heart dose in $\mathrm{AMBI}=0.93 \mathrm{~Gy}$ vs. median heart dose in A-PHDRBTboost $=1.8 \mathrm{~Gy}, p=0.01$ ) in left-breast treatments.

Finally, the MITBI-PHDRBT program allows administration of adjuvant radiotherapy as early as possible once the definitive pathological report is known. This characteristic reduces the duration of treatment significantly (five days of PHDRBT treatment, 11 days from BCS until the end of treatment in the AMBI group) compared to 25-30 days (60-70 days from BCS until the end of WBI in conventional treatment) or 15 days (40-50 days from BCS until the end of WBI with current standard hypofractionated regimen).

In view of the improvements in efficacy, safety, and logistics, which are possible with the MITBI-PHDRBT and AMBI program, it is feasible to explore ultra-accelerated schedules in properly selected low-risk patients. Despite encouraging results with mature follow-up documented in low-risk patients treated with AMBI, the main limitation of this study was the small number of patients included. Therefore, all the interpretations must be taken with a caution.

\section{Conclusions}

The MITBI-PHDRBT program allow selection of patients with favorable prognoses (G1-2 DCIS with negative margins and absence of multifocality), for whom AMBI could be a good alternative with low recurrence rate, decrease of unnecessary radiation, treatment logistics improvement, and over-treatment reduction. Patients with G3 DCIS on pre-operative biopsy are at more risk of reop- eration due to positive margins and worse local control; therefore the MITBI-PHDRBT for AMBI program is not recommended for these patients.

\section{Disclosure}

The authors report no conflict of interest.

\section{References}

1. Wapnir IL, Dignam JJ, Fisher B et al. Long-term outcomes of invasive ipsilateral breast tumor recurrences after lumpectomy in NSABP B-17 and B-24 randomized clinical trials for DCIS. J Natl Cancer Inst 2011; 103: 478-488.

2. Elshof LE, Schaapveld M, Schmidt MK et al. Subsequent risk of ipsilateral and contralateral invasive breast cancer after treatment for ductal carcinoma in situ: incidence and the effect of radiotherapy in a population-based cohort of 10,090 women. Breast Cancer Res Treat 2016; 159: 553-563.

3. Dunne C, Burke JP, Morrow M, Kell MR. Effect of margin status on local recurrence after breast conservation and radiation therapy for ductal carcinoma in situ. J Clin Oncol 2009; 27: 1615-1620.

4. Morrow M, Van Zee KJ, Solin LJ et al. Society of Surgical Oncology-American Society for Radiation Oncology-American Society of Clinical Oncology consensus guideline on margins for breast-conserving surgery with whole-breast irradiation in ductal carcinoma in situ. J Clin Oncol 2016; 34: 4040-4046.

5. Rath MG, Uhlmann L, Heil J et al. Predictors of residual tumor in breast-conserving therapy. Ann Surg Oncol 2015; 22: 451-458.

6. Donker $M$, Litière $S$, Werutsky $G$ et al. Breast-conserving treatment with or without radiotherapy in ductal carcinoma in situ: 15-year recurrence rates and outcome after a recurrence, from the EORTC 10853 randomized phase III trial. J Clin Oncol 2013; 31: 4054-4059.

7. McCormick B, Winter K, Hudis C et al. RTOG 9804: a prospective randomized trial for good-risk ductal carcinoma in situ comparing radiotherapy with observation. J Clin Oncol 2015; 33: 709-715.

8. Hong YK, McMasters KM, Egger ME, Ajkay N. Ductal carcinoma in situ current trends, controversies, and review of literature. Am J Surg 2018; 216: 998-1003.

9. van Seijen M, Lips EH, Thompson AM et al. Ductal carcinoma in situ: to treat or not to treat, that is the question. $\mathrm{Br}$ J Cancer 2019; 121: 285-292.

10. Barrio AV, Van Zee KJ. Controversies in the treatment of ductal carcinoma in situ. Ann Rev Med 2017; 68: 197-211.

11. Benitez PR, Streeter O, Vicini F et al. Preliminary results and evaluation of MammoSite balloon brachytherapy for partial breast irradiation for pure ductal carcinoma in situ: a phase II clinical study. Am J Surg 2006; 192: 427-433.

12. Israel PZ, Vicini F, Robbins AB et al. Ductal carcinoma in situ of the breast treated with accelerated partial breast irradiation using balloon-based brachytherapy. Ann Surg Oncol 2010; 17: 2940-2944.

13. Park SS, Grills IS, Chen PY et al. Accelerated partial breast irradiation for pure ductal carcinoma in situ. Int J Radiat Oncol Biol Phys 2011; 81: 403-408.

14. Jeruss JS, Kuerer HM, Beitsch PD et al. Update on DCIS outcomes from the american society of breast surgeons accelerated partial breast irradiation registry trial. Ann Surg Oncol 2011; 18: 65-71.

15. Abbott AM, Portschy PR, Lee C et al. Prospective multicenter trial evaluating balloon-catheter partial-breast irradiation for ductal carcinoma in situ. Int J Radiat Oncol Biol Phys 2013; 87: 494-498. 
16. Shah C, McGee M, Wilkinson J Ben et al. Clinical outcomes using accelerated partial breast irradiation in patients with ductal carcinoma in situ. Clin Breast Cancer 2012; 12: 259-263.

17. Correa C, Harris EE, Leonardi MC et al. Accelerated Partial Breast Irradiation: Executive summary for the update of an ASTRO Evidence-Based Consensus Statement. Pract Radiat Oncol 2017; 7: 73-79.

18. Strnad V, Major T, Polgar C et al. ESTRO-ACROP guideline: Interstitial multi-catheter breast brachytherapy as Accelerated Partial Breast Irradiation alone or as boost - GEC-ESTRO Breast Cancer Working Group practical recommendations. Radiother Oncol 2018; 128: 411-420.

19. Cox JD, Stetz JA, Pajak TF. Toxicity criteria of the Radiation Therapy Oncology Group (RTOG) and the European organization for research and treatment of cancer (EORTC). Int J Radiat Oncol Biol Phys 1995; 31: 1341-1346.

20. Wazer DE, DiPetrillo T, Schmidt-Ullrich R et al. Factors influencing cosmetic outcome and complication risk after conservative surgery and radiotherapy for early-stage breast carcinoma. J Clin Oncol 1992; 10: 356-363.

21. Veronesi U, Orecchia R, Maisonneuve P et al. Intraoperative radiotherapy versus external radiotherapy for early breast cancer (ELIOT): A randomised controlled equivalence trial. Lancet Oncol 2013; 14: 1269-1277.

22. Vaidya JS, Wenz F, Bulsara M et al. Risk-adapted targeted intraoperative radiotherapy versus whole-breast radiotherapy for breast cancer: 5-year results for local control and overall survival from the TARGIT-A randomised trial. Lancet 2014; 383: 603-613.

23. Coles CE, Griffin CL, Kirby AM et al. Partial-breast radiotherapy after breast conservation surgery for patients with early breast cancer (UK IMPORT LOW trial): 5-year results from a multicentre, randomised, controlled, phase 3, noninferiority trial. Lancet 2017; 390: 1048-1060.

24. Polgár C, Limbergen E Van, Pötter R et al. Patient selection for accelerated partial-breast irradiation (APBI) after breast-conserving surgery: Recommendations of the Groupe Européen de Curiethérapie-European Society for Therapeutic Radiology and Oncology (GEC-ESTRO) breast cancer working group based on clinical evidence (2009). Radiother Oncol 2010; 94: 264-273.

25. Strnad V, Ott OJ, Hildebrandt G et al. 5-year results of accelerated partial breast irradiation using sole interstitial multicatheter brachytherapy versus whole-breast irradiation with boost after breast-conserving surgery for low-risk invasive and in-situ carcinoma of the female breast: a randomised, phase 3, non-inferiority trial. Lancet 2016; 387: 229-238.

26. Vicini F, Shah C, Ben Wilkinson J et al. Should ductal carcinoma-in-situ (DCIS) be removed from the ASTRO consensus panel cautionary group for off-protocol use of accelerated partial breast irradiation (APBI)? A pooled analysis of outcomes for 300 patients with DCIS treated with APBI. Ann Surg Oncol 2013; 20: 1275-1281.

27. Bijker N, Meijnen P, Peterse JL et al. Breast-conserving treatment with or without radiotherapy in ductal carcinoma-in-situ: ten-year results of European Organisation for Research and Treatment of Cancer randomized phase III trial 10853 a study by the EORTC Breast Cancer Cooperative Group and EORTC Radiotherapy Group. J Clin Oncol 2006; 24: 3381-3387.

28. Wapnir IL, Dignam JJ, Fisher B et al. Long-term outcomes of invasive ipsilateral breast tumor recurrences after lumpectomy in NSABP B-17 and B-24 randomized clinical trials for DCIS. J Natl Cancer Inst 2011; 103: 478-488.

29. Leonardi MC, Corrao G, Frassoni $S$ et al. Ductal carcinoma in situ and intraoperative partial breast irradiation: Who are the best candidates? Long-term outcome of a single institution series. Radiother Oncol 2019; 133: 68-76. 